\title{
Contemporary Cardiac SPECT Imaging- Innovations and Best Practices: An Information Statement from the American Society of Nuclear Cardiology
}

\author{
Brian G. Abbott, MD, MASNC, 'James A. Case, PhD, FASNC, ' Sharmila Dorbala, \\ MD, MPH, FASNC, ${ }^{c}$ Andrew J. Einstein, MD, PhD, FASNC, ${ }^{\mathrm{d}}$ James R. Galt, PhD, ${ }^{\mathrm{e}}$ \\ Robert Pagnanelli, BSRT(R)(N), CNMT, NCT, ${ }^{f}$ Renée P. Bullock-Palmer, MD, \\ FASNC, ${ }^{\mathrm{g}}$ Prem Soman, MD, PhD, FASNC, ${ }^{\mathrm{h}}$ and R. Glenn Wells, PhD ${ }^{\mathrm{i}}$ \\ a Warren Alpert Medical School, Brown University, Providence, RI \\ b Cardiovascular Imaging Technologies, Kansas City, MO \\ c Harvard Medical School, Brigham and Women's Hospital, Boston, MA \\ d Columbia University Irving Medical Center and New York-Presbyterian Hospital, New York, NY \\ e Emory University School of Medicine, Atlanta, GA \\ f Duke University Medical Center, Durham, NC \\ g Department of Cardiology, Deborah Heart and Lung Center, Browns Mills, NJ \\ h Department of Medicine, University of Pittsburgh, Pittsburgh, PA \\ i University of Ottawa Heart Institute, Ottawa, Canada
}

doi: $10.1007 / \mathrm{s} 12350-018-1348-y$

This information statement from the American Society of Nuclear Cardiology highlights advances in cardiac SPECT imaging and supports the incorporation of new technology and techniques in laboratories performing nuclear cardiology procedures. The document focuses on the application of the latest imaging protocols and the utilization of newer hardware and software options to perform high quality, state-of-the-art SPECT nuclear cardiology procedures. Recommendations for best practices of cardiac SPECT imaging are discussed, highlighting what imaging laboratories should be doing as the standard of care in 2018 to achieve optimal results (based on the ASNC 2018 SPECT guideline [Dorbala et al., J Nucl Cardiol. 2018. https://doi.org/10.1007/s12350-018-1283-y]).

Co-publication: This article is being jointly published in the Journal of Nuclear Cardiology and Circulation: Cardiovascular Imaging.

Disclaimer: The opinions expressed in this article are not necessarily those of the editors or of the American Heart Association.

Reprint requests: Brian G. Abbott, MD, MASNC, Warren Alpert Medical School, Brown University, Providence, RI; babbott@lifespan.org
J Nucl Cardiol 2018;25:1847-60.

1071-3581/ $\$ 34.00$

Copyright (C) 2018 American Society of Nuclear Cardiology and American Heart Association, Inc. 


\begin{tabular}{|ll|}
\hline Abbreviations \\
CAD & Coronary artery disease \\
CT & Computed tomography \\
CZT & Cadmium zinc telluride \\
FBP & Filtered backprojection \\
FFR & Fractional flow reserve \\
MBF & Myocardial blood flow \\
MPI & Myocardial perfusion imaging \\
OSEM & Ordered subset expectation \\
& maximization \\
PET & Positron emission tomography \\
SPECT & Single Photon Emission Computed \\
& Tomography \\
\hline
\end{tabular}

\section{INTRODUCTION}

Myocardial perfusion imaging (MPI) performed using Single Photon Emission Computed Tomography (SPECT) remains the most common procedure in nuclear cardiology, owing to a robust database of clinical studies demonstrating invaluable efficacy and utility in the evaluation and risk stratification of patients with known or suspected cardiovascular disease. While cardiac SPECT technology has been in widespread use clinically for the past three decades, recent advances in equipment and protocols aimed at optimizing image quality, improving diagnostic accuracy, and reducing radiation exposure have not been widely incorporated into existing practice. ${ }^{1,2}$

The American Society of Nuclear Cardiology (ASNC) recently published updates to two SPECT Imaging Guidelines, one addressing "Instrumentation, Acquisition, Processing, and Interpretation", 3 and the other addressing "Stress, Protocols, and Tracers". 4 These documents apprise providers of nuclear cardiology procedures of standards for their optimal performance. This document highlights the use of new technology and protocols addressed in these recommendations. Given recent advances in Cardiac SPECT imaging, the aim of the current document is to both highlight these innovations and also provide guidance on best practices for performing high quality imaging, employing a patient-centered approach, to achieve optimal results that will have a meaningful impact on patient management and outcomes.

\section{HARDWARE}

Most SPECT systems in use today are based on technology that has been in use for more than 25 years. These are dual-detector systems, with parallel-hole collimation and sodium-iodide (NaI) scintillation cameras mounted at $90^{\circ}$ to each other. This configuration allows acquisition greater than $180^{\circ}\left(45^{\circ}\right.$ leftanterior- oblique to $45^{\circ}$ left-posterior oblique) with a gantry rotation of only $90^{\circ}$. Two configurations are available: The first have detectors fixed at $90^{\circ}$ and are dedicated to nuclear cardiology. The second have large field-of-view detectors that may be used in a $90^{\circ}$ configuration for cardiac imaging or with the detectors at $180^{\circ}$ (or other angles) for general nuclear medicine. While outwardly the systems may not have changed much since their introduction, advances in reconstruction algorithms and processing techniques have greatly improved performance, as discussed later in this document.

More recently introduced systems have increased photon sensitivity through the use of high-sensitivity collimation and multiple detectors to perform a cardiocentric or heart-centered acquisitions (See Figure 1). Regardless of the collimation used, each system also takes advantage of advanced SPECT reconstruction algorithms to further improve the sensitivity of the system. ${ }^{5,6}$

\section{New Technology for High-Sensitivity SPECT}

Two such systems utilize direct-conversion, solidstate detectors made of cadmium zinc telluride (CZT) but very different collimation schemes. The main advantage of CZT as a detector is that it has much better energy resolution than $\mathrm{NaI}$ scintillation and can be used to construct compact pixelated detector modules, where each pixel (a single CZT crystal $2.46 \mathrm{~mm}$ on each side) is smaller than the intrinsic resolution of a NaI scintillation camera. Clinical CZT systems achieve energy resolution of better than $7 \%$, compared to about $11 \%$ for a conventional scintillation camera. Both systems use detectors based on modular units of 16-X16 CZT pixels but in different arrangements.

The first CZT system uses nine rectangular detectors with very high sensitivity parallel-hole collimation that sweep across the heart of the patient $\left(\mathrm{D}-\mathrm{SPECT}^{\circledR}\right.$, Spectrum Dynamics Medical). Each rectangular detector is constructed of four modules arranged in a column (16x-64 pixels). Collimators are made of tungsten, with square holes designed to match the detector pixels. During acquisition, each of the nine detectors simultaneously sweeps across the patients thorax. Counts from the heart are maximized by confining the sweep of each detector to the region of the heart. An initial clinical trial of the system with Tc-99m radiopharmaceuticals concluded that D-SPECT acquisitions of 4 and 2 minutes for MPI stress and rest studies yielded studies that correlated well with 16- and 12-minutes studies acquired 

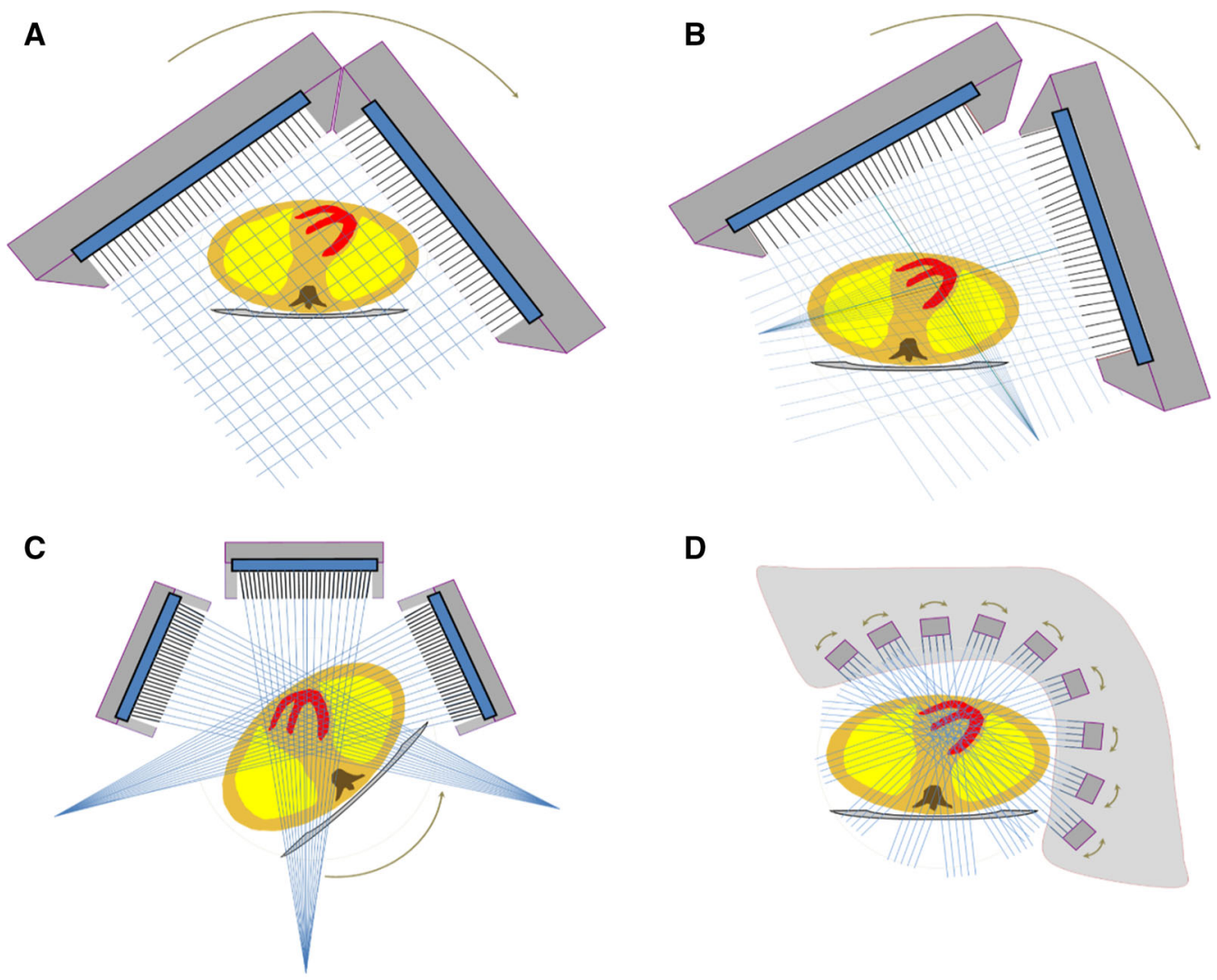

D

$\mathbf{E}$

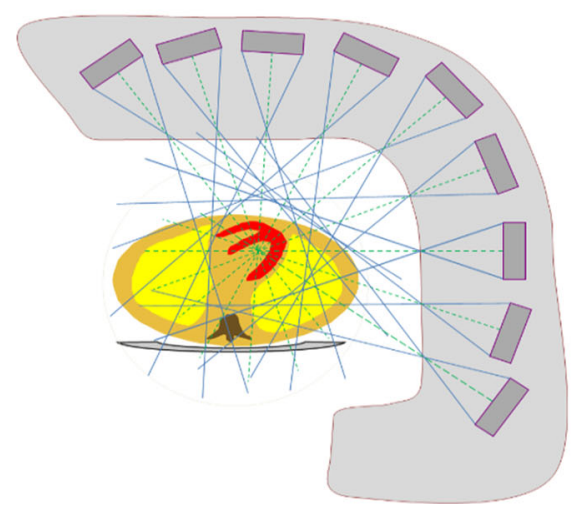

Figure 1. SPECT System Configurations. (A) Conventional dual-detector SPECT with parallelhole collimation. (B) IQ·SPECT utilizes unique collimation on a conventional dual-detector SPECT system to maximize sensitivity over the heart while avoiding collimation of the body. (C) Cardius-3 SPECT system with three CsI pixelated scintillation cameras. The patient rotates for acquisition, while the detectors remain stationary. (D) D-SPECT with nine columnar CZT detectors that simultaneously sweep across the heart during acquisition. (E) Discovery 530c with 19 pinhole detectors all directed at the heart. Two rows of five detectors; each are adjacent to the row of nine detectors in the diagram and provide views of the heart from above and below the transaxial plane. The detectors and the patient are stationary during acquisition.

with conventional SPECT and had equivalent diagnostic performance. $^{7}$

The second CZT system uses 19 square CZT detectors, each equipped with a single tungsten pinhole collimator (Discovery 530c, GE Healthcare). Each detector is comprised of four modules (32-x-32 pixels). The detector are arranged in three rows, with the center row of nine detectors perpendicular to the patients 
thorax. Two adjacent rows of detectors (five each) are oriented so they image from above and below the center line (giving a three-dimensional acquisition). The center line of each of the 19 pin-hole collimators passes through the same point in space. With this system, the detectors and the patient remain stationary during acquisition, and the heart of a properly positioned patient is centered on the point where the center lines of all the pin-hole collimators meet. A multicenter trial of the system with a 1 day technetium- $99 \mathrm{~m}$ tetrofosmin rest/stress MPI of 4 and 2 minutes compared well with 14- and 12-minutes acquisitions with conventional SPECT systems. ${ }^{8}$

The Cardius SPECT systems (Digirad Corp.) use pixelated scintillation detectors. Each cesium-iodide (CsI) scintillation detector is matched to a silicon photodiode that detects scintillation in the crystal. Eliminating the photomultiplier tubes used in traditional systems allows the camera to be considered a solid-state device and the design of more compact detectors. In this system, the patient sits upright in a chair that rotates. When equipped with two detectors and parallel-hole collimation, system sensitivity should be comparable to a traditional dual-detector SPECT system. A multicenter trial of another configuration, using three detectors to increase sensitivity and resolution recovery reconstruction, demonstrated that a 5-minutes rest acquisition and a 4-minutes stress acquisition could yield perfusion and function information from gated SPECT myocardial perfusion imaging studies that were diagnostically equivalent to full-time acquisition and standard reconstruction. ${ }^{9}$ Further improvements in sensitivity can be had by replacing the parallel-hole collimators with fanbeam collimators, maximizing counts from the heart. ${ }^{10}$

One system, IQ.SPECT, achieves high sensitivity by using a unique collimator design on a standard, large field-of-view, dual-detector SPECT or SPECT/CT system (IQ.SPECT, Siemens Healthineers). ${ }^{11}$ The confocal collimators used in IQ.SPECT have a central area with converging collimation designed to focus on the heart and transition to parallel-hole collimation around the periphery of the camera. This design allows increased sensitivity over the heart where it is most needed but avoids truncation of the body. ${ }^{11}$ The use of IQ.SPECT to reduce imaging time and/or radiopharmaceutical dose has been demonstrated in phantoms and in patients..$^{11,12}$ Lyon et al compared AC stress SPECT to IQ.SPECT using a dose of $925-1100 \mathrm{MBq}(25-30 \mathrm{mCi}) \mathrm{Tc}-99 \mathrm{~m}$ sestamibi. Several different count levels were simulated for IQ.SPECT and evaluated using system and countlevel specific normal files. The study concluded that IQ.SPECT could be used to reduce both the dose and the time by half compared to conventional SPECT. Thus the standard dose could be reduced to below $550 \mathrm{MBq}$
$(15 \mathrm{mCi})$, and the imaging time reduced from $13 \mathrm{~min}-$ utes (standard SPECT) to 7 minutes (IQ.SPECT). ${ }^{12}$ Only when the confocal collimator is mounted is the system restricted to nuclear cardiology. When equipped with other collimators, the system is a general purpose SPECT or SPECT/CT, a factor that may appeal to clinics that also perform general nuclear medicine imaging studies.

\section{SOFTWARE}

\section{SPECT Processing Techniques}

Many of the perceived shortcomings of myocardial perfusion SPECT imaging can be traced to the use of outdated image processing software. Because these solutions can be implemented without a large capital investment, every laboratory should review their processing software and identify the opportunities to modernize.

\section{Optimal Reconstruction}

In the early days of SPECT imaging, limitations on computing power, memory, and disk space left few options for reconstructing the tomographic slices. Filtered backprojection (FBP) reconstruction had been the primary reconstruction algorithm since the beginning of SPECT imaging. This approach, though common, is prone to image artifacts, such as the ramp filter artifact, ${ }^{13}$ noise amplification ${ }^{14,15}$ and cannot be used for non-uniform attenuation correcting or improving resolution. ${ }^{16,17}$ This has a direct impact on the accuracy of SPECT interpretation. It also requires the use of radiation doses much higher than what is used in other modalities for similar procedures. ${ }^{18,19}$

Iterative reconstruction algorithms utilize a stepwise approach to determine the source distribution; the most common of these is the maximum likelihood/expectation maximization (MLEM) algorithm. ${ }^{20}$ This algorithm uses a model for the statistics of the image to iteratively improve the source activity estimate. The primary advantage of iterative reconstruction algorithms is that they use a model of the entire imaging process to create the tomographic slices. This model can be customized to include compensation for almost any physical process: attenuation, scatter, collimator blur, noise, etc. ${ }^{21-23}$

Though this algorithm is robust, adaptable, and produces good-quality images, it can be computationally intensive when applied to gated and dynamic data. More recently, the ordered subsets, expectation maximization algorithm has been used to accelerate the reconstruction times of the MLEM algorithm. ${ }^{24}$ This acceleration 


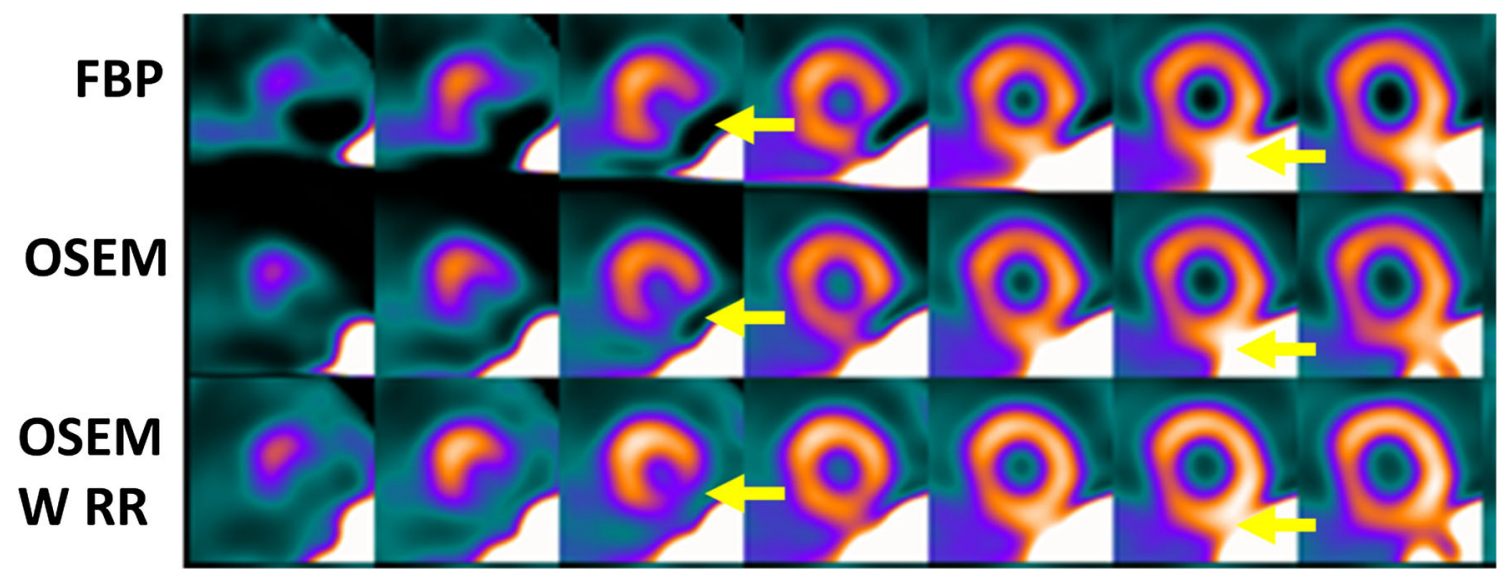

Figure 2. The ramp-filter artifact can reduce the counts in regions near high uptake. Iterative reconstruction approaches, such as OSEM and OSEM with resolution recovery (OSEM w RR) can reduce or eliminate this artifact.

technique reduces reconstruction times by more than ten times, making iterative reconstruction practical on modern personal computers.

Use of iterative reconstruction and resolution recovery can improve the signal to noise and reduce common reconstruction artifacts. Comparisons of ordered subset expectation maximization with resolution recovery (OSEM-RR) reconstructions with FBP demonstrate that up to an 8-fold improvement in signal to noise can be obtained when OSEM-RR is used. ${ }^{15}$ In addition, ramp filter artifacts from gut activity can also be reduced (see Figure 2). ${ }^{13}$

Because of the improvements to signal to noise and reduction of artifacts, such as the ramp filter and the widespread availability of upgrade paths for legacy processing workstations, clinical practices should identify pathways for implementing iterative reconstruction.

\section{Advanced Iterative Reconstruction for Reducing Patient dose and Acquisition Time}

Many commercial software solutions are available for improving the resolution of the reconstructed images. $^{25-28}$ These technologies are easily integrated on existing Anger Camera SPECT imaging systems. This improvement in resolution has the effect of boosting the signal coming from the myocardium. This boost in signal can be used to reduce the required dose and or acquisition time of the study. Given the widespread availability of these solutions and the advantages to patient comfort and reducing patient dose, nuclear cardiology labortories should investigate pathways to include resolution recovery into daily practice.

\section{Advanced Iterative Reconstruction for Implementing Attenuation Correction}

Correction for soft-tissue attenuation has been shown to improve specificity, improve reader confidence, and reduce the need for rest imaging in stressonly protocols. ${ }^{29}$ To implement attenuation correction, the iterative reconstruction algorithm can be adapted to include compensation for attenuation based on a patientspecific attenuation map.

When implementing attenuation correction, software should allow for the assessment of the quality of both the attenuation map and transmission/emission registration. In addition, attenuation correction performs best when combined with other corrections, such as resolution recovery and scatter correction. SPECT attenuation is combined with other corrections, such as scatter and collimator resolution correction, and the final image quality is similar to positron emission tomography (PET) (see Figure 3). ${ }^{30}$ Because of the potential to improve test accuracy and image quality, practices should consider attenuation correction hardware when replacing legacy SPECT instrumentation.

\section{ATTENUATION COMPENSATION}

The interpretation and quantification of myocardial perfusion SPECT are hampered by photon attenuation, Compton scatter, and collimator resolution effects. These factors preclude a linear relationship between the counts in the image and the true tracer distribution. The application of attenuation correction has been demonstrated to improve overall test accuracy, reduce difference between male and female studies, improve the assessment of large body-mass-index (BMI) patients 


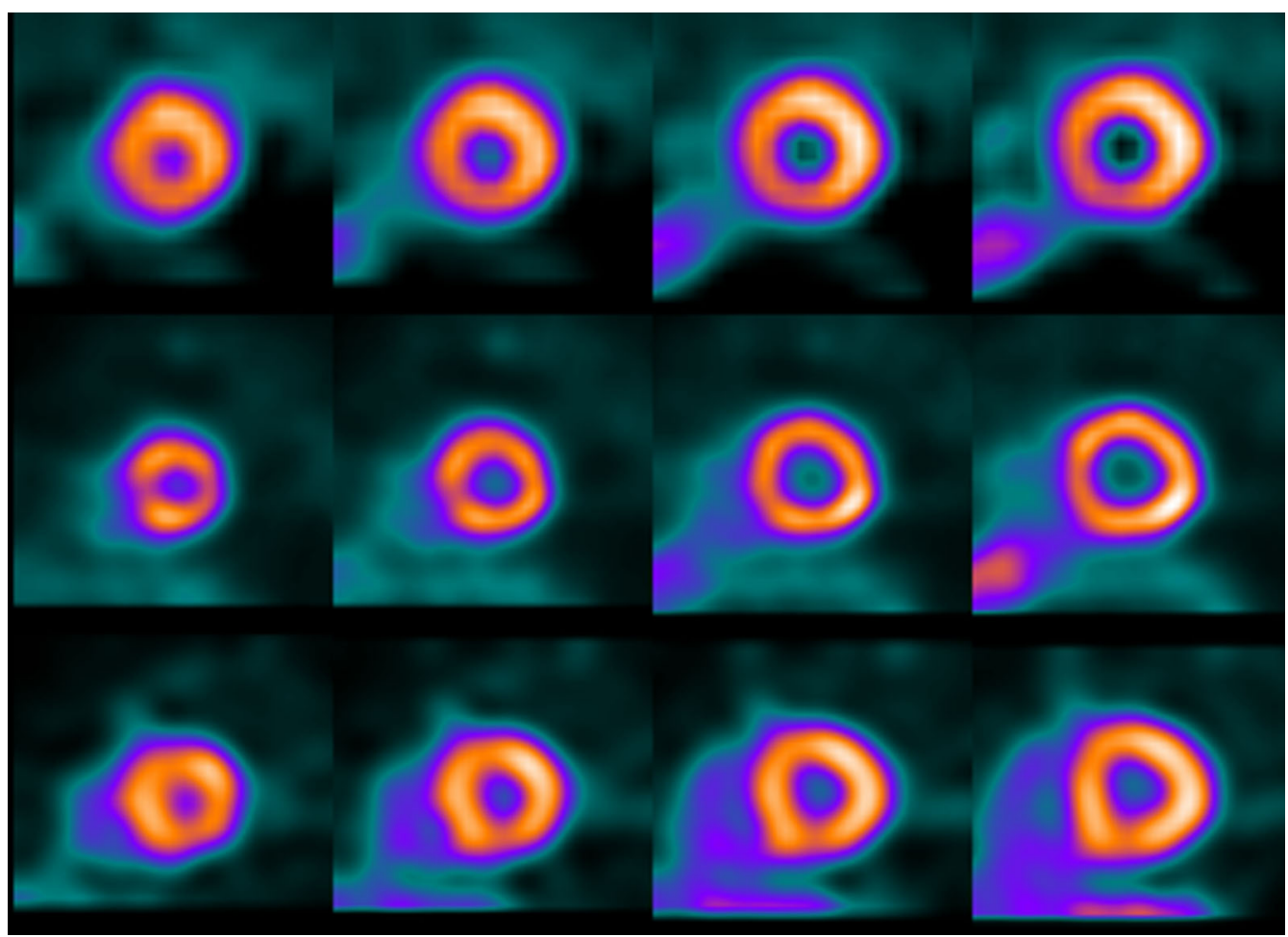

Figure 3. Comparison of two different SPECT reconstruction algorithms of a 9-mCi Tc-99msestamibi study at rest: Top row is FBP; middle row is OSEM with attenuation, scatter, and collimator resolution correction. Bottom row is a myocardial perfusion PET study with $25 \mathrm{mCi}$ of $\mathrm{Rb}-82$ at rest in the same patient.

and improving the usefulness of stress only imaging. ${ }^{31-36}$

Attenuation correction may be performed when a patient-specific map of anatomical attenuation is available. This is typically acquired using a transmission scan, either from scanning radionuclide sources or CT). Alternately, it is often possible to determine if an apparent myocardial defect is an artifact by performing a second SPECT scan with the body in a different position, altering the attenuation pattern. ${ }^{37-39}$

Attenuation affects cardiac SPECT images in both easily identifiable and subtle ways. Artifacts associated with breast attenuation in women and diaphragmatic attenuation in men are familiar to those proficient with conventional SPECT systems. ${ }^{40,41}$ Recognition of these artifacts is complicated because association with gender is not exclusive, and the position and extent may vary greatly. Exaggerated diaphragmatic attenuation can occur in women, and significant pectoral musculature or gynecomastia can yield artifacts in men similar to those that are more expected in women. Images produced by new, high-sensitivity SPECT systems are also subject to attenuation artifacts. The artifacts may not follow the familiar patterns, however, because of the position of the patient during imaging (particularly if the images are acquired with the patient in a sitting position) or the geometry of the SPECT system (particularly if the system uses multiple cameras to surround the patient).

Attenuation correction for myocardial perfusion SPECT has been recommended by ASNC for several years but has not been universally adopted by the nuclear cardiology community. ${ }^{42,43}$ Reasons for this include the added expense and complexity of the transmission scan, the sizable investment needed for SPECT/CT, and lack of reimbursement. There is also a lack of general awareness of the improved diagnostic accuracy and interpretive confidence afforded by the addition of attenuation correction. Computed tomography scanner-based attenuation also has the added value of assessing for the presence of coronary artery calcification on the transmission of CT images to detect subclinical coronary artery disease (CAD). ${ }^{44}$

If attenuation correction hardware is not available, sequential scans with the body in two different positions can be used to distinguish attenuation artifacts from perfusion defects, and thereby compensate for attenuation artifacts. For example, when the body's position is moved to prone from supine, the heart's position within the body changes, and attenuators - such as the breasts and diaphragm-shift. The pattern of attenuation is 
different and a defect that is present with the body in one position, but not the other, is unlikely to be due to an actual defect and can be considered artifact. ${ }^{37,38}$ Acquiring the prone images in addition to supine images adds complexity and increases the time required to acquire each patient's images. Two-position imaging has become more practical as new reconstruction techniques have allowed the use of shorter scan times.

When nuclear cardiology laboratories are upgrading nuclear instrumentation, SPECT/CT should be considered given its importance in reducing image artifacts, stress-only imaging and coronary calcium assessment when SPECT/CT. If attenuation correction is not available, 2-position imaging is encouraged to reduce equivocal and false-positive results. Regardless of the attenuation-compensation method used, reducing the uncertainly of attenuation in clinical images improved the confidence of the clinician in the images and is of particular importance when stress-only protocols are used because, by eliminating attenuation artifacts, they reduce the need for resting images. ${ }^{36,39,45,46}$

\section{Equipment for Attenuation Correction}

Application of attenuation correction requires a patient-specific map of the attenuating media, the attenuation map. The most practical way of measuring the attenuation map is through a transmission scan, either made with a radionuclide sealed source or through CT. Many early attenuation correction methods were based on transmission scans made with sealed sources of activity. Several different methods have been commercially released, with the most common being a line source that scanned across the camera field of view in each planar projection. This allows the same camera that acquires the emission scan to acquire the transmission scan. Use of Gd-153 with an apparent energy peak at $100 \mathrm{keV}$ as the source allowed discrimination between the transmission photons and the emission photons from the heart (140 keV for Tc-99m based radiopharmaceuticals). Reconstruction of the transmission scan uses the same algorithms as used in CT imaging.

In the last ten years, the availability of SPECT/CT systems has made the use of radionuclide-based transmission systems less common. Hybrid SPECT/CT systems incorporate a CT scanner into the SPECT gantry. Two types of systems have been released. Slowrotation, low-power systems utilize the SPECT gantry to rotate both the gamma cameras and the CT assembly. ${ }^{47}$ The first SPECT/CT system released incorporated this type of system and produced $1-\mathrm{cm}$ thick slices with a typical transmission scan for attenuation correction taking about 5 minutes. Improvements of this system cut the slice thickness to $0.5 \mathrm{~cm}$ and the imaging time for attenuation correction to about 2.5 minutes. More recently a system using a flat-panel detector has been introduced. This allows a CT scan of the thorax containing the myocardium to be acquired with one spin of the gantry (about 20 seconds). The second configuration for SPECT/CT incorporates a standarddesign CT system in tandem with the SPECT in a similar design to most PET/CT systems. In all of the SPECT/CT configurations, the SPECT and CT are acquired sequentially and care must be taken to avoid movement of the body that would cause misregistration of the SPECT/CT (see Figure 4). All, SPECT/CT studies should be inspected for misregistration and corrected when necessary.

It should be noted that all of the methods discussed above increase the radiation dose to the patient. This is minimal $(1-2 \mu \mathrm{Sv})$ for the sealed source systems due to the low photon flux. ${ }^{48}$ Tandem SPECT/CT systems often incorporate clinical CT systems and are capable of delivering a relatively high radiation dose. ${ }^{49}$ If the sole use of the CT scan is attenuation correction, the CT parameters (tube current $[\mathrm{mA}]$, tube potential $[\mathrm{kV}$ or $\mathrm{kVp}$, rotation speed, and pitch) should be chosen to minimize the radiation dose. Doses below $1 \mathrm{mSv}$ can produce acceptable attenuation correction and can be visually assessed for coronary calcifications. ${ }^{44}$

Ideally the patient's body should be in the exact same position in the SPECT and CT scans. In practice, the duration of the CT scan is much shorter than the SPECT, and the patient's breathing pattern can impact the registration of the SPECT and CT. The standard practice for a chest CT might be an inspiration breathhold, but this guarantees that the shape of the body is very different between the two scans. ASNC guidelines recommend the patient be instructed to breath shallowly (free tidal breathing) or to hold their breath after exhaling, ${ }^{50}$ either of which will produce a better match than inspiration. After acquisition of SPECT and CT, the registration should be checked and corrected if necessary (see Figure 4).

Just as extra-cardiac uptake of the radiopharmaceutical should be reported if the clinician believes that it may indicate a problem, the CT scan should be examined for abnormalities and, if found, reported. With CT scanner-based attenuation, however, there is the added value of assessing for the presence of coronary artery calcification on the transmission $\mathrm{CT}$ images to detect subclinical CAD. ${ }^{44}$

\section{Attenuation Compensation with New Technology}

In the case of the high-sensitivity SPECT systems, attenuation compensation is available either through use 
A

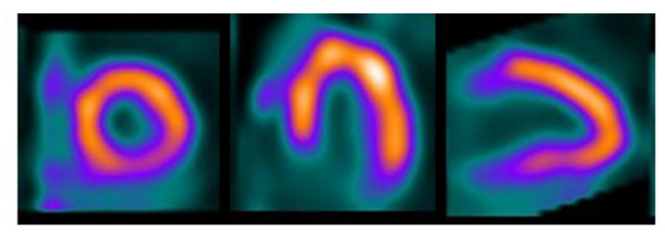

B
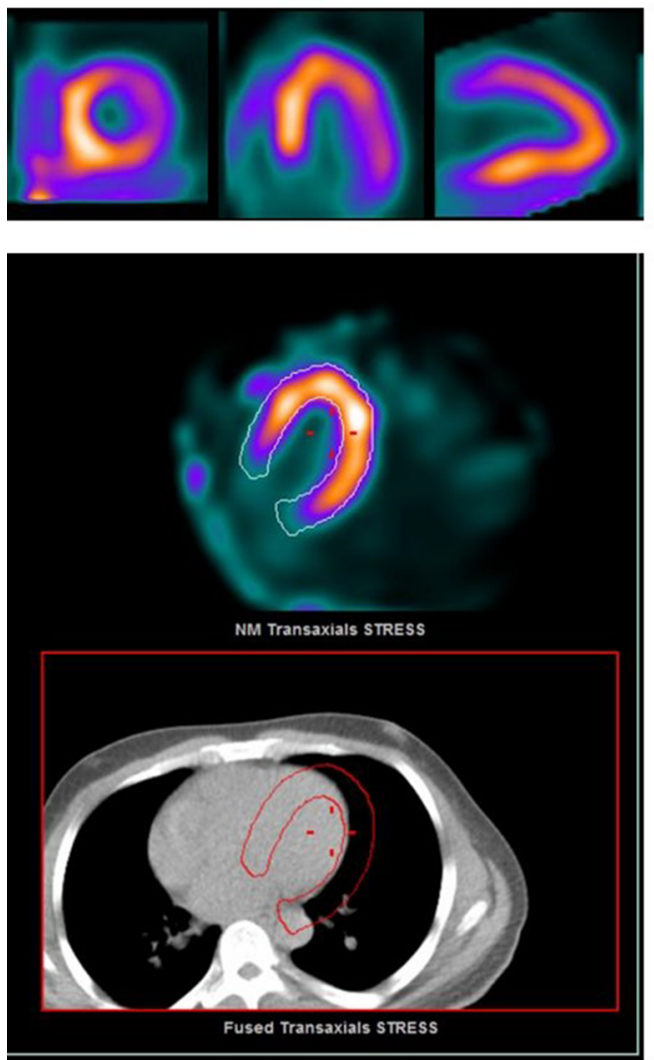

C
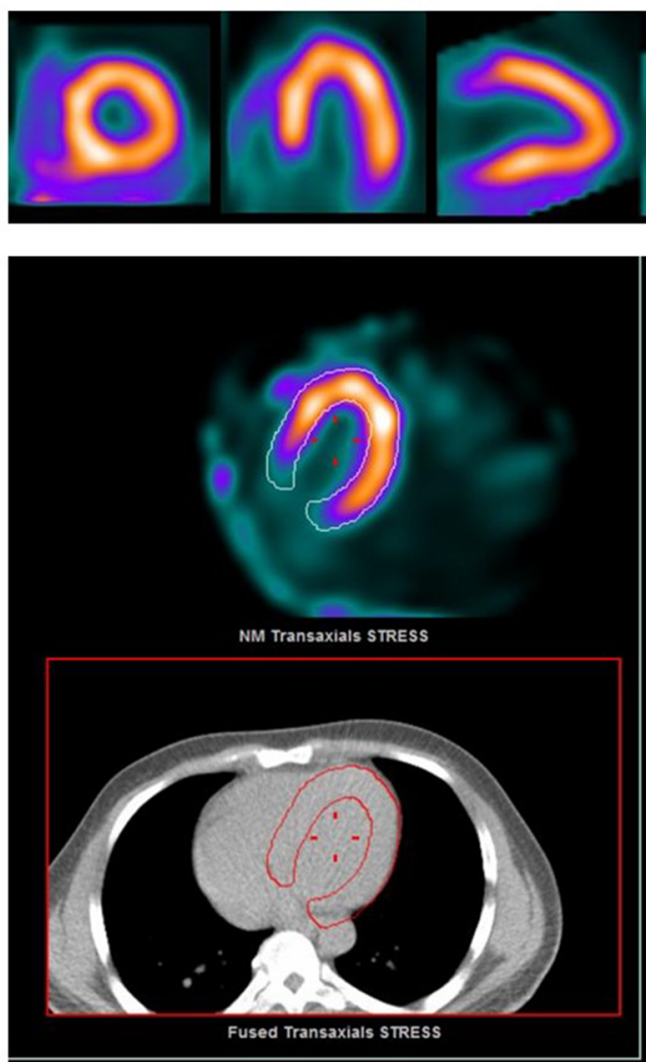

Figure 4. Accurate alignment of SPECT and CT is required for accurate CT-based attenuation correction. (A) Tc-99m-tetrofosmin stress study $(6 \mathrm{mCi})$ acquired on a Discovery 530c SPECT system and reconstructed without attenuation correction. (B) Poor registration where SPECT myocardial tissue is overlaid on CT lung, resulting in under correction of attenuation in the lateral wall. (C) When properly aligned, the attenuation correction produces myocardial uptake that appears uniform.

of attenuation compensation with SPECT/CT or by twoposition imaging.

The D-SPECT system (Spectrum Dynamics) does not have the capability of attenuation correction, but it does allow the patient to be imaged in two positions. In this system, the patient sits in a chair and is nearly upright in standard imaging. The patient may also be reclined to a supine position and a second image acquired. As with supine/prone imaging, a defect that is evident with the body in one position but not seen with a body in another position is likely to be artifact. ${ }^{51,52}$
The GE Discovery 530c (GE Healthcare) was initially available in a SPECT/CT configuration utilizing a 64-slice CT scanner. The system also has an option to import a CT from a separate scanner for use in attenuation correction. Regardless of the source, the SPECT and the CT scans are registered prior to attenuation correction. Attenuation correction with this system may help improve the quantitative specificity without a loss in sensitivity. ${ }^{53}$ Prone imaging is also available and has been shown to help identify patient's at low risk for death or myocardial infarction. ${ }^{54}$ 
X-ACT (Digirad Corp.) is a novel CT system where a target rod is bombarded with $\mathrm{X}$-rays from a tube. Characteristic X-rays produced after photoelectric absorption in the target rod are used for the transmission scan instead of the output of the X-ray tube. This results in mono-energetic photons for the transmission imaging, which can be more accurately transformed into attenuation coefficients for attenuation correction than the Hounsfield units produced by the broad-spectrum photons produced directly from the X-ray tube. The transmission scan is acquired using the same detectors as the emission scan. ${ }^{10}$

IQ.SPECT (Siemens Medical Solutions) is based on a conventional dual-detector SPECT scanner and is available as a SPECT/CT. Some reports have indicated that the system performs best if attenuation correction is used. ${ }^{55}$ Other investigators have shown that a combined supine/prone imaging protocol can also identify attenuation artifacts in supine images. 56

\section{OPTIMIZING STRESS TESTING PROTOCOLS WITH SPECT}

A same-day, low-dose stress first followed by optional rest-imaging protocol yields the lowest radiation-dose exposure-as low as $1 \mathrm{mSv}$ with the stress only protocol on a high-sensitivity (solid-state) system. ${ }^{57}$ Patient comfort is improved along with radiation dose savings to laboratory staff and cost savings to the practice when using unit dose radiotracers. The use of attenuation correction or 2-position imaging (additional prone or upright imaging) is helpful to decrease attenuation artifacts and decrease the need for rest acquisition in many cases. The findings of a recent study suggest that stress first protocol also may minimize shine through of rest activity and improve the detection of ischemia. $^{58} \mathrm{~A}$ normal stress first imaging has been shown to portend excellent prognosis, comparable to prognostic value of normal rest/stress imaging. Stress first has prognostic proven value in several subsets of patients, including men and women, patients with or without diabetes, obese vs non obese patients. ${ }^{59}$ Further evaluation with angiography (CT or invasive) may be considered despite normal stress first imaging, if the stress test shows high risk features, left ventricular ejection fraction is reduced, or patient has ongoing symptoms. ${ }^{3}$ The use of low-dose stress-first protocol is important and essential to perform low radiation dose MPI to minimize the radiation-dose exposure to the patient, especially if the patient has no history of prior myocardial infarction or reduced left ventricular systolic function.

The improved resolution and count sensitivity of newer camera hardware technology for SPECT MPI, as well as novel iterative reconstruction software for processing, has translated into lower radiotracer dose requirements for stress testing, with the development of low-dose protocols. This has resulted in significantly reduced radiation exposure with studies achieving less than $7 \mathrm{mSv}$ of effective radiation dose. ${ }^{60}$ The new technology also permits shorter acquisition times, which can significantly reduce the potential for motion artifacts, particularly in patients with orthopedic and/or musculoskeletal limitations. As such, individualized or "patient-centered" protocols rather than a "one-sizefits-all" approach are recommended, including 2-position imaging to reduce the rates of equivocal studies due to attenuation from the diaphragm or breast/anterior chest wall adipose tissue.

Weight-based radiotracer dosing and/or increased acquisition time should be routinely considered for SPECT imaging studies, tailored to the patient so as to optimize radiation dose given the patient's habitus, while minimizing the likelihood of poor image quality due to insufficient cardiac counts or to patient motion. In addition to the single-day or two-day standard dose protocols with either stress first, followed by optional rest-image acquisition, or rest first followed by stressimage acquisition, there have been newer protocols that have been recommended with low-dose imaging (see Figure 5). With the shorter scan times associated with newer technology, the use of 2-position imaging to shift attenuators has become more practical.

It is recommended that low-dose imaging be considered for most patients presenting for stress testing in laboratories that have newer software or hardware technology available. For patients with a high body mass index, low-dose imaging may be limited due to poor count statistics resulting in poor image quality, and a longer acquisition time (with newer software/hardware, and cooperative patients) or two-day equal dose protocol (conventional hardware/software) may be considered to lower radiation dose.

New software and hardware technology also allows for faster scan times, such as half-time imaging, with acquisition times as short as 5 to 7.5 minutes (see Figure 5), and should be considered for patients unable to tolerate standard time imaging.

\section{BEST PRACTICE RECOMMENDATIONS}

Among choices of SPECT technologies (see Figure 6), while iterative reconstruction is generally preferable to FBP, reconstruction software adding noise reduction and resolution recovery to iterative reconstruction is preferable to iterative reconstruction alone, and use of a solid-state high-efficiency camera incorporating this software is an ideal choice for many patients. 


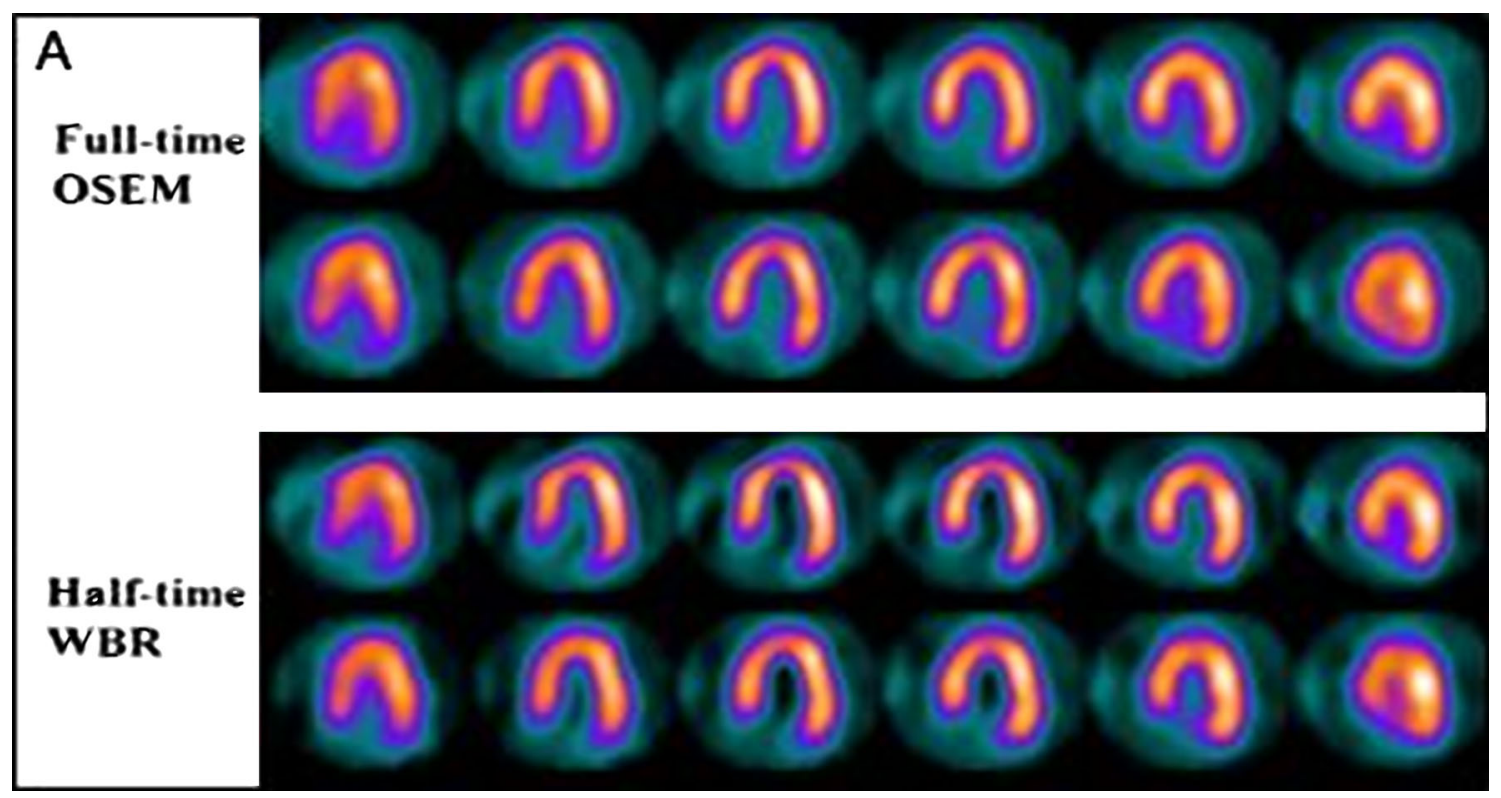

Figure 5. Case example of half-time, wide-beam reconstruction acquisition. ${ }^{3}$

Use of single-position uncorrected images, while most common for SPECT MPI, is not ideal, but rather use of attenuation compensation, by means of attenuation correction technology or attenuation shifting with twoposition imaging (e.g., supine/prone or sitting/semirecumbent), is a preferred practice. Computed tomography-based attenuation correction is the ideal approach to address attenuation for many patients. Among SPECT protocol options, no one choice is right for all patients; however, dual isotope imaging is not recommended, whereas single-day low- or ultra-lowdose stress-first/optional rest protocols offer the opportunity to minimize radiation exposure.

Thus, for many patients, the combined use of novel SPECT software and hardware, low-dose stress first (optional rest) with 2-position imaging serves as an ideal combination of technology and protocol to achieve the best diagnostic images and improved sensitivity while minimizing the radiation dose. For other patients, use of a hybrid SPECT/CT camera with CT-based attenuation correction and estimation of calcium score, often combined with a low-dose stress-first imaging protocol, is the optimal choice. No one protocol is optimal for all patients and practice settings.

\section{INTERPRETATION STRATEGIES}

The interpretation of images acquired on any of the high-sensitivity SPECT systems requires special considerations. ${ }^{3,55,61-64}$ Familiar clues to attenuation and motion artifacts may not be available on systems without standard collimator or projection images. Frequently encountered attenuation patterns may produce myocardial perfusion images significantly different from standard SPECT systems, particularly with upright imaging. The resolution of the system, the collimator geometry, and proprietary reconstruction algorithms may produce differences as well. Thus, a "recalibration" of the interpretive eye is required, and can be achieved with experience. Furthermore, both attenuation correction and the 2-position approach to attenuation artifact recognition require that the interpreter integrate two images for comparison (attenuation correction/Nonattenuation correction, supine/prone, upright/supine). Each of the software packages available for interpretation has facilities that support these comparisons.

\section{FUTURE DIRECTIONS: MYOCARDIAL BLOOD FLOW QUANTIFICATION WITH SPECT}

The traditional approach to myocardial perfusion assessment by SPECT is limited in fundamental ways: First, it is relative, in that regional tracer uptake is normalized to the myocardial segment with the highest uptake; therefore, global flow reduction may not be recognized, and although the technique is highly accurate to detect obstructive $\mathrm{CAD}$, the extent of CAD is generally underestimated. Second, performance of the Tc-99m-based flow tracers-and to a lesser extent Tl201 - is limited by a low myocardial extraction fraction resulting in a roll-off of tracer uptake at higher flow rates in the range encountered during vasodilator stress 


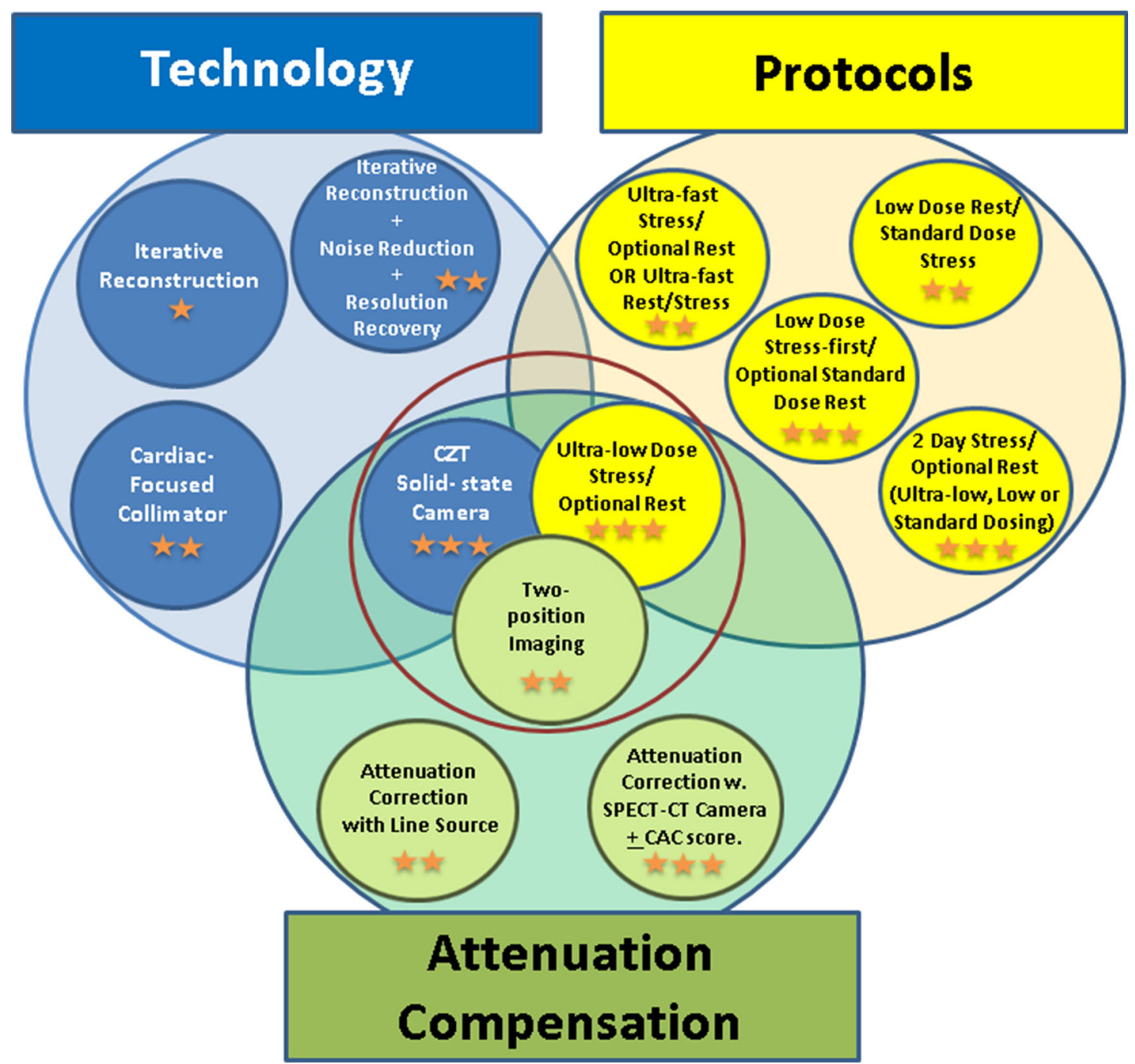

Figure 6. Venn diagram showing innovative software and hardware technology, study protocols, and attenuation compensation methods available for SPECT. Number of stars reflects relative overall levels of innovation within a category; nevertheless, for an individual patient, an option with fewer stars may be preferable or more practical. $C Z T$ denotes cadmium-zinc-telluride. CAC denotes coronary artery calcium. Ultra-low Dose here refers to Newer technology reduced-dose protocols, specified in the bottom half of Table 5 of ASNC Stress, Protocols, and Tracers. ${ }^{4}$ Ultra-fast here refers to reduced-acquisition-time protocols for Newer technology. For lower risk, younger patients, a combination of an ultra-low dose protocol on a CZT camera with two-position imaging, shown circled in red in the center, may represent the approach best optimizing the use of innovative approaches. For patients with high BMI, or complicated coronary disease, optimization may favor attenuation correction and iterative reconstruction software incorporating noise reduction and resolution recovery.

testing, which reduces contrast between ischemic and normal myocardium. ${ }^{65}$ Absolute myocardial blood flow (MBF) quantification using dynamic imaging can obviate these limitations. This has been easier to implement in PET due to high count statistics, high temporal resolution tomographic imaging and robust attenuation correction combined with the availability of PET perfusion tracers with excellent extraction- characteristics, like N-13 ammonia, O-15 water. Flow quantification with myocardial perfusion SPECT using a traditional Anger scintillation camera has been difficult due to the challenge of obtaining very rapid, tomographic images of the inflow and outflow of tracer activity during the first pass. In addition, accurate 
estimation of myocardial blood flow in SPECT is complicated by the low first-pass extraction fraction of Tc- $99 \mathrm{~m}$ perfusion tracers. The introduction of cardiac cameras based on solid-state detector technology and new tomographic acquisition approaches have made rapid dynamic acquisition of the input bolus possible. ${ }^{63,66-68}$ However, the lack of attenuation correction with contemporary solid-state SPECT systems, and the relatively poor extraction fraction of current SPECT flow tracers, continue to pose challenges.

Although data on MBF measurement using SPECT in humans are limited at present, SPECT MBF with Tc$99 \mathrm{~m}$ sestamibi has been shown to correlate with disease severity measured with relative perfusion imaging and to differentiate non-obstructive from obstructive disease measured with fractional flow reserve (FFR) ${ }^{69}$ Studies of SPECT MBF with Tc-99m tetrofosmin ${ }^{70}$ and Tl$201^{71}$ have also shown good correlation with the presence of multi-vessel disease measured with FFR and invasive coronary angiography. Other studies have demonstrated good correlations $(\mathrm{r} \geq 0.83)$ between SPECT MBF and PET MBF measured with ammonia or rubidium, and water. $^{63,68,72}$ Further studies are needed, however, to demonstrate the incremental diagnostic and prognostic benefit of SPECT MBF over relative perfusion imaging alone. At the current time, more than $95 \%$ of radionuclide perfusion studies are still performed with SPECT; therefore, the ability to perform dynamic SPECT could be a significant enhancement to the field of nuclear cardiology.

\section{Summary}

Myocardial perfusion imaging performed with SPECT remains an invaluable tool in the evaluation and management in patients with known or suspected CAD. Incorporating recent advances in stress imaging protocols, camera technology, and processing software can enhance diagnostic accuracy, as well as reduce radiation exposure. Providers of nuclear cardiology procedures should strive to integrate as many of these innovations in their laboratories as possible to achieve optimal imaging results.

\section{Disclosures}

Sharmila Dorbala, MD, MPH, FASNC has received grant support from Astellas Pharma, had investments in General Electric, and has served as a consultant for Bracco Diagnostics and for GE Healthcare. Andrew Einstein, MD, PhD, FASNC receives grant support from Toshiba America Medical Systems and has served as a consultant to GE Healthcare. James $R$. Galt, PhD receives royalties paid through Emory University for Emory QuantEM software for analysis of renal scintigraphy. Robert Pagnanelli, BSRT(R)(N), CNMT, NCT, FASNC serves on the Speakers' Bureau and Advisory Board for Astellas Pharma US. Prem Soman, MD, PhD, FASNC receives research grant support from Astellas Pharma and serves on the Advisory Board for Alnylam Pharmaceuticals. $R$. Glenn Wells, PhD receives honoraria from GE Healthcare and grant support from GE Healthcare and Advanced Accelerator Applications. All other contributors have nothing relevant to disclose.

\section{References}

1. American Society of Nuclear Cardiology/MedAxiom Nuclear Survey. J Nucl Cardiol 2014;21:5-88. https://doi.org/10.1007/s12 350-014-9862-z.

2. Einstein AJ, Pascual TN, Mercuri M, Karthikeyan G, Vitola JV, Mahmarian JJ, et al. Current worldwide nuclear cardiology practices and radiation exposure: Results from the 65 country IAEA Nuclear Cardiology Protocols Cross-Sectional Study (INCAPS). Eur Heart J 2015;36:1689-96. https://doi.org/10.1093/eurheartj/eh v117.

3. Dorbala S, Ananthasubramaniam K, Armstrong IS, Chareonthaitawee P, DePuey EG, Eisntein AJ, et al. Single Photon Emission Computed Tomography (SPECT) myocardial perfusion imaging guidelines: Instrumentation, acquisition, processing, and interpretation. J Nucl Cardiol 2018. https://doi.org/10.1007/s1235 0-018-1283-y.

4. Henzlova MJ, Duvall WL, Einstein AJ, Travin MI, Verberne HJ. ASNC imaging guidelines for SPECT nuclear cardiology procedures: Stress, protocols, and tracers. J Nucl Cardiol 2016;23:60639. https://doi.org/10.1007/s12350-015-0387-x.

5. Slomka PJ, Berman DS, Germano G. New cardiac cameras: Single-photon emission CT and PET. Semin Nucl Med 2014;44:23251.

6. Piccinelli M, Garcia EV. Advances in single-photon emission computed tomography hardware and software. Cardiol Clin 2016;34:1-11.

7. Sharir T, Ben-Haim S, Merzon K, Prochorov V, Dickman D, BenHaim $S$, et al. High-speed myocardial perfusion imaging: Initial clinical comparison with conventional dual detector anger camera imaging. JACC Cardiovasc Imaging 2008;1:156-63. https://doi. org/10.1016/j.jcmg.2007.12.004.

8. Esteves FP, Raggi P, Folks RD, Keidar Z, Askew JW, Rispler S, et al. Novel solid-state-detector dedicated cardiac camera for fast myocardial perfusion imaging: Multicenter comparison with standard dual detector cameras. J Nucl Cardiol 2009;16:927-34.

9. Maddahi J, Mendez R, Mahmarian JJ, Thomas G, Babla H, Bai C, et al. Prospective multicenter evaluation of rapid, gated SPECT myocardial perfusion upright imaging. J Nucl Cardiol 2009;16:351-7.

10. Bai C, Conwell R, Kindem J, Babla H, Gurley M, De Los Santos $\mathrm{R}$, et al. Phantom evaluation of a cardiac SPECT/VCT system that uses a common set of solid-state detectors for both emission and transmission scans. J Nucl Cardiol 2010;17:459-69.

11. Caobelli F, Kaiser SR, Thackeray JT, Bengel FM, Chieregato M, Soffientini A, et al. IQ SPECT allows a significant reduction in administered dose and acquisition time for myocardial perfusion imaging: Evidence from a phantom study. J Nucl Med 2014;55:2064-70

12. Lyon MC, Foster C, Ding X, Dorbala S, Spence D, Bhattacharya $\mathrm{M}$, et al. Dose reduction in half-time myocardial perfusion 
SPECT-CT with multifocal collimation. J Nucl Cardiol 2016;23:657-67. https://doi.org/10.1007/s12350-016-0471-x.

13. DePuey EG. Advances in SPECT camera software and hardware: Currently available and new on the horizon. J Nucl Cardiol 2012;19:551-81.

14. Wilson DW, Tsui BMW. Noise properties of filtered-backprojection and ML-EM reconstructed emission tomographic images. IEEE Trans Nucl Sci 1993;40:1198-203.

15. Case JA, Jensen J, Courter SA, Helmuth P, Bateman TM. Improved quantitative SPECT myocardial uniformity of myocardial uptake using half-time iterative resolution recovery algorithm. J Nucl Cardiol 2017;1481 [abstract].

16. King MA, Tsui BMW, Pan T-S, Glick SJ, Soares EJ. Attenuation compensation for cardiac single-photon emission computed tomographic imaging: Part 2. Attenuation compensation algorithms. J Nucl Cardiol 1996;3:55-64.

17. Glick SJ, Penney BC, King MA, Byrne CL. Noniterative compensation for the distance-dependent detector response and photon attenuation in SPECT imaging. IEEE Trans Med Imaging 1994;13:363-74.

18. Hill KD, Einstein AJ. New approaches to radiation exposure. Trends Cardiovasc Med 2016;26:55-65.

19. Case JA, DeKemp RA, Slomka PJ, Smith MF, Heller GV, Cerqueira MD. Status of cardiovascular PET radiation exposure and strategies for reduction: An Information Statement from the Cardiovascular PET Task Force. J Nucl Cardiol 2017;24:1427-39.

20. Shepp LA, Vardi Y. Maximum likelihood reconstruction for emission tomography. IEEE Trans Med Imaging 1982;1:113-21.

21. Zeng GL, Gullberg GT, Tsui BMW, Terry JA. Three-dimensional iterative reconstruction algorithms with attenuation and geometric point response correction. IEEE Trans Nucl Sci 1991;38:693-702.

22. Hebert T, Leahy R. A generalized EM algorithm for 3-D Bayesian reconstruction from Poisson data using Gibbs priors. IEEE Trans Med Imaging 1989;8:194-202.

23. Liang Z, Turkington TG, Gilland DR, Jaszczak RJ, Coleman RE. Simultaneous compensation for attenuation, scatter, and detector response for SPECT reconstructions in three dimensions. Phys Med Biol 1992;37:587-603.

24. Hudson HM, Larkin RS. Accelerated image reconstruction using ordered subsets of projection data. IEEE Trans Med Imaging 1994;13:601-9.

25. Kadrmas DJ, Frey EC, Karimi SS, Tsui BM. Fast implementations of reconstruction-based scatter compensation in fully 3D SPECT image reconstruction. Phys Med Biol 1998;43:857-73.

26. Borges-Neto S, Pagnanelli RA, Shaw LK, Honeycutt E, Shwartz SC, Adams GL, et al. Clinical results of a novel wide beam reconstruction method for shortening scan time of Tc-99m cardiac SPECT perfusion studies. J Nucl Cardiol 2007;14:555-65.

27. Druz RS, Phillips LM, Chugkowski M, Boutis L, Rutkin B, Katz S. Wide-beam reconstruction half-time SPECT improves diagnostic certainty and preserves normalcy and accuracy: A quantitative perfusion analysis. J Nucl Cardiol 2011;18:52-61

28. Venero CV, Heller GV, Bateman TM, McGhie AI, Ahlberg AW, Katten D, et al. A multicenter evaluation of a new post-processing method with depth-dependent collimator resolution applied to fulltime and half-time acquisitions without and with simultaneously acquired attenuation correction. J Nucl Cardiol 2009;16:714-25.

29. Heller GV, Bateman TM, Johnson LL, Cullom SJ, Case JA, Galt $\mathrm{JR}$, et al. Clinical value of attenuation correction in stress-only Tc99m sestamibi SPECT imaging. J Nucl Cardiol 2004;11:273-81.

30. Bateman TM, Heller GV, McGhie AI, Courter SA, Golub RA, Case JA, et al. J Multicenter investigation comparing a highly efficient half-time stress-only attenuation correction approach against standard rest-stress Tc-99m SPECT imaging. J Nucl Cardiol 2009;16:726-35.

31. Hendel RC, Berman DS, Cullom SJ, Follansbee W, Heller GV, Kiat $\mathrm{H}$, et al. Multicenter clinical trial to evaluate the efficacy of correction for photon attenuation and scatter in SPECT myocardial perfusion imaging. Circulation 1999;99:2742-9.

32. Ficaro EA, Fessler JA, Shreve PD, Kritzman JN, Rose PA, Corbett JR. Simultaneous transmission/emission myocardial perfusion tomography: Diagnostic accuracy of attenuation corrected Tc-99m sestamibi single-photon emission computed tomography. Circulation 1996;93:463-73.

33. Duvernoy CS, Ficaro EP, Karabajakian MZ, Rose PA, Corbett JR. Improved detection of left main coronary artery disease with attenuation-corrected SPECT. J Nucl Cardiol 2000;7:639-48.

34. Thompson RC, Heller GV, Johnson LL, Case JA, Cullom SJ, Moutray KL, et al. Value of attenuation correction on ECG-gated SPECT myocardial perfusion imaging related to body mass index. J Nucl Cardiol 2005;12:195-202.

35. Grossman GB, Garcia EV, Bateman TM, Heller GV, Johnson LL, Folks RD, et al. Quantitative technetium-99m sestamibi attenuation corrected SPECT: development and multicenter trial validation of myocardial perfusion stress gender-independent normal database in an obese population. $\mathrm{J}$ Nucl Cardiol 2004; 11:263-72.

36. Mathur S, Heller GV, Bateman TM, Ruffin R, Yekta A, Katten D, et al. Clinical value of stress-only Tc-99m SPECT imaging: Importance of attenuation correction. J Nucl Cardiol 2013;20:2737.

37. Segall GM, Davis MJ. Prone vs supine thallium myocardial SPECT: A method to decrease artifactual inferior wall defects. J Nucl Med 1989;30:548-55.

38. Cohen MC. Combined supine and prone imaging acquisition in cardiac SPECT: A turn for the better. J Nucl Cardiol 2016;23:1477-9.

39. Gutstein A, Bental T, Solodky A, Mats I, Zafrir N. Prognosis of stress-only SPECT myocardial perfusion imaging with prone imaging. J Nucl Cardiol 2016.

40. DePuey EG, Garcia EV. Optimal specificity of thallium-201 SPECT through recognition of imaging artifacts. J Nucl Med 1989;30:441-9.

41. DePuey EG. How to detect and avoid myocardial perfusion SPECT artifacts. J Nucl Med 1994;35:699-702.

42. Hendel RC, Corbett JR, Cullom SJ, DePuey EG, Garcia EV, Bateman TM. The value and practice of attenuation correction for myocardial perfusion SPECT imaging: A joint position statement from the American Society of Nuclear Cardiology and the Society of Nuclear Medicine. J Nucl Cardiol 2002;9:135-43.

43. Cerqueira MD, Allman KC, Ficaro EP, Hansen CL, Nichols KJ, Thompson RC, et al. Recommendations for reducing radiation exposure in myocardial perfusion imaging. J Nucl Cardiol 2010;17:709-18.

44. Einstein AJ, Johnson LL, Bokhari S, Son J, Thompson RC, Bateman TM, et al. Agreement of visual estimation of coronary artery calcium from low-dose CT attenuation correction scans in hybrid PET/CT and SPECT/CT with standard Agatston score. J Am Coll Cardiol 2010;56:1914-21.

45. van Dijk JD, Mouden M, Ottervanger JP, van Dalen JA, Knollema $\mathrm{S}$, Slump $\mathrm{CH}$, et al. Value of attenuation correction in stress-only myocardial perfusion imaging using CZT-SPECT. J Nucl Cardiol 2017;24:395-401. https://doi.org/10.1007/s12350-015-0374-2.

46. Jameria ZA, Abdallah M, Fernandez-Ulloa M, O'Donnell R, Dwivedi AK, Washburn E, et al. Analysis of stress-only imaging, comparing upright and supine CZT camera acquisition to conventional gamma camera images with and without attenuation 
correction, with coronary angiography as a reference. J Nucl Cardiol 2018;25:540-9. https://doi.org/10.1007/s12350-017-07817.

47. Seret A, Nguyen D, Bernard C. Quantitative capabilities of four state-of-the-art SPECT-CT cameras. EJNMMI Res 2012;2:45.

48. Perisinakis K, Theocharopoulos N, Karkavitsas N, Damilakis J. Patient effective radiation dose and associated risk from transmission scans using $153 \mathrm{Gd}$ line sources in cardiac spect studies. Health Phys 2002;83:66-74.

49. Chen J, Einstein AJ, Fazel R, Krumholz HM, Wang Y, Ross JS, et al. Cumulative exposure to ionizing radiation from diagnostic and therapeutic cardiac imaging procedures: A population-based analysis. J Am Coll Cardiol 2010;56:702-11.

50. Dorbala S, Di Carli MF, Delbeke D, Abbara S, DePuey EG, Dilsizian V, et al. SNMMI/ASNC/SCCT guideline for cardiac SPECT/CT and PET/CT 1.0. J Nucl Med 2013;54:1485-507.

51. Nakazato R, Tamarappoo BK, Kang X, Wolak A, Kite F, Hayes SW, et al. Quantitative upright-supine high-speed SPECT myocardial perfusion imaging for detection of coronary artery disease: Correlation with invasive coronary angiography. J Nucl Med 2010;51:1724-31.

52. Ben-Haim S, Almukhailed O, Neill J, Slomka P, Allie R, Shiti D, et al. Clinical value of supine and upright myocardial perfusion imaging in obese patients using the D-SPECT camera. J Nucl Cardiol 2014;21:478-85.

53. Esteves FP, Galt JR, Folks RD, Verdes L, Garcia EV. Diagnostic performance of low-dose rest/stress Tc-99m tetrofosmin myocardial perfusion SPECT using the 530c CZT camera: Quantitative vs visual analysis. J Nucl Cardiol 2014;21:158-65.

54. Worden NE, Lindower PD, Burns TL, Chatterjee K, Weiss RM. A second look with prone SPECT myocardial perfusion imaging reduces the need for angiography in patients at low risk for cardiac death or MI. J Nucl Cardiol 2015;22:115-22.

55. Gremillet E, Agostini D. How to use cardiac IQ.SPECT routinely? An overview of tips and tricks from practical experience to the literature. Eur J Nucl Med Mol Imaging 2016;43:707-10.

56. Takamura T, Horiguchi Y, Kanna M, Matsushita H, Sudo Y, Kikuchi S, et al. Validation of prone myocardial perfusion SPECT with a variable-focus collimator vs supine myocardial perfusion SPECT with or without computed tomography-derived attenuation correction. Ann Nucl Med 2015;29:890-6.

57. Einstein AJ, Johnson LL, DeLuca AJ, Kontak AC, Groves DW, Stant $\mathrm{J}$, et al. Radiation dose and prognosis of ultra-low-dose stress-first myocardial perfusion SPECT in patients with chest pain using a high-efficiency camera. J Nucl Med 2015;56:545-51. h ttps://doi.org/10.2967/jnumed.114.150664.

58. Thomas GS, Cullom SJ, Kitt TM, Feaheny KM, Ananthasubramaniam K, Gropler RJ, et al. The EXERRT trial: EXErcise to Regadenoson in Recovery Trial: A phase 3b, open-label, parallel group, randomized, multicenter study to assess regadenoson administration following an inadequate exercise stress test as compared to regadenoson without exercise for myocardial perfusion imaging using a SPECT protocol. J Nucl Cardiol 2017;24:788-802. https://doi.org/10.1007/s12350-017-0813-3.

59. Chang SM, Nabi F, Xu J, Raza U, Mahmarian JJ. Normal stressonly vs standard stress/rest myocardial perfusion imaging: Similar patient mortality with reduced radiation exposure. J Am Coll Cardiol 2010;55:221-30. https://doi.org/10.1016/j.jacc.2009.09. 022 .
60. DePuey EG, Ata P, Wray R. $5 \mathrm{mCi}$ stress myocardial perfusion SPECT with a conventional NaI camera. J Nucl Med. 2012;53:1792.

61. Timmins R, Ruddy TD, Wells RG. Patient position alters attenuation effects in multipinhole cardiac SPECT. Med Phys 2015;42:1233-40.

62. Allie R, Hutton BF, Prvulovich E, Bomanji J, Michopoulou S, Ben-Haim S. Pitfalls and artifacts using the D-SPECT dedicated cardiac camera. J Nucl Cardiol 2016;23:301-10. https://doi.org/10. 1007/s12350-015-0277-2.

63. Agostini D, Roule V, Nganoa C, Roth N, Baavour R, Parienti JJ, et al. First validation of myocardial flow reserve assessed by dynamic 99mTc-sestamibi CZT-SPECT camera: Head to head comparison with 150-water PET and fractional flow reserve in patients with suspected coronary artery disease. The WATERDAY study: Eur J Nucl Med Mol Imaging.; 2018. https://doi.org/10. 1007/s00259-018-3958-7.

64. Hindorf C, Oddstig J, Hedeer F, Hansson MJ, Jögi J, Engblom H. Importance of correct patient positioning in myocardial perfusion SPECT when using a CZT camera. J Nucl Cardiol 2014;21:695-702.

65. Soman P, Taillefer R, DePuey EG, Udelson JE, Lahiri A. Enhanced detection of reversible perfusion defects by Tc-99m sestamibi compared to Tc-99m tetrofosmin during vasodilator stress SPECT imaging in mild-to-moderate coronary artery disease. J Am Coll Cardiol 2001;37:458-62.

66. Gambhir SS, Berman DS, Ziffer J, Nagler M, Sandler M, Patton J, et al. A novel high-sensitivity rapid-acquisition single-photon cardiac imaging camera. J Nucl Med 2009;50:635-43. https://doi. org/10.2967/jnumed.108.060020.

67. Wells RG, Timmins R, Klein R, Lockwood J, Marvin B, deKemp RA, et al. Dynamic SPECT measurement of absolute myocardial blood flow in a porcine model. J Nucl Med 2014;55:1685-91. h ttps://doi.org/10.2967/jnumed.114.139782.

68. Wells RG, Marvin B, Poirier M, Renaud JM, deKemp RA, Ruddy TD. Optimization of SPECT measurement of myocardial blood flow with corrections for attenuation, motion, and blood-binding compared to PET. J Nucl Med 2017;58:2013-9. https://doi.org/10. 2967/jnumed.117.191049.

69. Ben-Haim S, Murthy VL, Breault C, Allie R, Sitek A, Roth N, et al. Quantification of myocardial perfusion reserve using dynamic SPECT imaging in humans: A feasibility study. J Nucl Med 2013;54:873-9.

70. Ben Bouallegue F, Roubille F, Lattuca B, Cung TT, Macia JC, Gervasoni R, et al. SPECT myocardial perfusion reserve in patients with multivessel coronary disease: Correlation with angiographic findings and invasive fractional flow reserve measurements. J Nucl Med 2015;56:1712-7. https://doi.org/10.2967/ jnumed.114.143164.

71. Shiraishi S, Sakamoto F, Tsuda N, Yoshida M, Tomiguchi S, Utsunomiya D, et al. Prediction of left main or 3-vessel disease using myocardial perfusion reserve on dynamic thallium-201 single-photon emission computed tomography with a semiconductor gamma camera. Circ J 2015;79:623-31. https://doi.org/10. 1253/circj.CJ-14-0932.

72. Nkoulou R, Fuchs TA, Pazhenkottil AP, Kuest SM, Ghadri JR, Stehli J, et al. Absolute myocardial blood flow and flow reserve assessed by gated SPECT with cadmium-zinc-telluride detectors using 99mTc-Tetrofosmin: Head-to-head comparison with $13 \mathrm{~N}$ ammonia PET. J Nucl Med 2016;57:1887-92. 